\title{
Mice with Mutation in Dynein Heavy Chain 1 Do Not Share the Same Tau Expression Pattern with Mice with SOD1-Related Motor Neuron Disease
}

\author{
Magdalena Kuźma-Kozakiewicz • Ewa Usarek • \\ Albert C. Ludolph • Anna Barańczyk-Kuźma
}

Accepted: 24 February 2011/Published online: 6 March 2011

(C) The Author(s) 2011. This article is published with open access at Springerlink.com

\begin{abstract}
Due to controversy about the involvement of Dynclhl mutation in pathogenesis of motor neuron disease, we investigated expression of tau protein in transgenic hybrid mice with Dynclhl (so-called Cra1/+), SOD1G93A (SOD1/+), double (Cra1/SOD1) mutations and wild-type controls. Total tau-mRNA and isoforms 0,1 and $2 \mathrm{~N}$ expression was studied in frontal cortex, hippocampus, spinal cord and cerebellum of presymptomatic and symptomatic animals (age 70, 140 and 365 days). The most significant differences were found in brain cortex and cerebellum, but not in hippocampus and spinal cord. There were less changes in Cra1/SOD1 double heterozygotes compared to mice harboring single mutations. The differences in total tau expression and in profile of its isoforms between Cra1/+ and SOD1/+ transgenics indicate a distinct pathogenic entity of these two conditions.
\end{abstract}

Keywords Tau Motor neuron disease $\cdot$ Dynein . Superoxide dismutase- $1 \cdot$ Mutations $\cdot$ Mice hybrids

M. Kuźma-Kozakiewicz

Department of Neurology, Medical University of Warsaw, Banacha 1a, 02097 Warsaw, Poland

E. Usarek · A. Barańczyk-Kuźma ( $₫)$

Department of Biochemistry, Medical University of Warsaw,

Banacha 1, 02097 Warsaw, Poland

e-mail: anna.kuzma@wum.edu.pl

A. C. Ludolph

Department of Neurology, University of Ulm, Oberer Eselsberg

45, 89081 Ulm, Germany

\section{Introduction}

Intracellular transport along microtubules is especially important in motor neurons, the axons of which may reach the length of over one meter [1]. The major components of this transport are microtubules, actin filaments and motor proteins. Many studies indicate an impairment of axonal transport in motor neuron diseases. Cytoplasmic dynein is one of microtubule associated proteins (MAPs) responsible for axonal transport toward the minus end of microtubules $[2,3]$. It is a large multi-subunit protein complex (MW about 1.5 MD) formed of two identical heavy chains (MW $532 \mathrm{kD}$ ), few intermediate chains (MW 74 and 53-59 kD), and several light chains [4]. Heavy chains bind ATP, a source of energy for dynein movement along microtubules and the intermediate chains probably anchor dynein to its cargo. Heavy chains are encoded by a 78 -exon gene, Dynclhl. Knocking out the Dynclhl results in early embryonic lethality in mice [5]. Mice with an autosomal dominant missense point mutations in the Dnchcl gene show a progressive limb paresis. Due to abnormal body twisting and cramping of the hindlimbs when suspended by tail they were called Legs at odd angles (Loa) and Cramping 1 (Cra1) mice. On molecular level Cra1 mice show defects in dynein complex resulting in an impaired retrograde axonal transport [6-8]. The exchange of tyrosine to cysteine (Cra1: 1051VWLQCQCLW1059; wild type: 1051VWLQYQCLW1059) within the dynein heavy chain leads to an age related progressive degeneration of the motor neurons. The phenotype of Cra1 mice is similar (but milder) to transgenic mice with SOD1G93A mutation, recognized as the animal model of amyotrophic lateral sclerosis [9]. In contrast to SOD1 mice, the life span of Cra1 and Loa heterozygotes remains almost unchanged compared to wild-type animals [8]. In motor neurons of 
SOD1G93A mice, the defects in retrograde axonal transport appear already during embryonic development. Unexpectedly, crossing SOD1G93A mice with Loa or Cra1 delays the disease progression and significantly increases the life span of SOD1 double heterozygotes (Loa/SOD1 and Cra1/SOD1) [10]. Thus, mutations in the Dnclhl gene appear to influence the progression of motor neuron degeneration and SOD1G93A-toxicity [10, 11]. However, there are publications that call in question the above opinion [12-15]. Dupuis et al. (2009) proved that Cra/+ heterozygotes do not display typical feature of motor neuron disease, even in aged animals, but instead they develop an early-onset sensory neuropathy.

Beside the main motor proteins, including dynein/dynactin complex and kinesins, there are other motors involved in the axonal transport. Among them, the tau protein, responsible for polymerization and stabilization of microtubules in neurons, astrocytes and oligodendrocytes, as well as their organization into bundles [16-18]. Tau also enhances the attachment of dynein/dynactin complex to microtubules by binding proper $\mathrm{N}$-terminal part to the C-terminus of the 150 subunit of dynactin [19]. The mutation of the latter was found responsible for the motor neuron disease in humans [20]. Human tau gene contains 16 exons, but tau primary transcript present in the central nervous system consists of 13 exons [21-23]. Exons 2, 3 and 10 undergo alternative splicing giving rise to six different mRNAs translated into six different protein isoforms. These isoforms differ from each other by the presence or absence of three inserts encoded by exons 2 and 3 at the $\mathrm{N}$-terminal part, and exon 10 at the C-terminal part. The $0 \mathrm{~N}$ tau isoform does not contain products of exons 2 and $3(-2-3)$, whereas isoform $1 \mathrm{~N}$ contains an insert encoded by exon $2(+2-3)$, and isoform $2 \mathrm{~N}$ an insert encoded by exons 2 and $3(+2+3)$ [21]. The product of exon 10 (R2 domain) is present at the C-terminal of tau in adults brain giving rise to a $4 \mathrm{R}(+10)$ isoform. $4 \mathrm{R}$ is absent from mammals fetal brain, where the $3 \mathrm{R}(-10)$ isoform is present [24]. In contrast to rodents, in adult humans both isoforms (4R and 3R) are present in equal amounts [25].

Due to controversy considering the involvement of dynein heavy chain 1 mutation in pathogenesis of motor neuron degeneration, in the present study we compared the changes in the tau alternative mRNA splicing and in the total tau expression between the SOD1G93A transgenic mice and the transgenic hybrid mice with the Dynclhl (Cra1/+) and double Dynclhl/SOD1G93A mutations (Cra1/SOD1).

\section{Experimental Procedure}

Mouse strain C57BL/6GJ-C3H/HeJ (B6-C3H) hybrids harboring human $\mathrm{Cu} / \mathrm{Zn}$ superoxide dismutase gene with
G93A amyotrophic lateral sclerosis-associated mutation (SOD1/+), hybrids with mutation in dynein heavy chain 1 (Dync1h1; Cra1/+), and with double Dync1h1/SOD1G93A mutation (Cra1/SOD1) were bred at the animal facility of the University of Ulm, Germany. The SOD1G93A males (B6 background; Jackson Laboratories, Ben Harbor, ME, USA) were crossed with Cra1/+ heterozygote female mice (C3He background; Ingenium Pharmaceuticals AG, Martinsried, Germany), as described earlier by Teuchert et al. (2006). Animals were at age of 70 days (presymptomatic stages of the diseases for SOD1/+ and Cra1/SOD1 groups), 140 days (presymptomatic stage for Cra1/+ and symptomatic for SOD1/+ and Cra1/SOD1), and 365 days (symptomatic stage for Cra1/+). Age- and genetic background-matched wild-type hybrid mice $(+/+)$ were used as controls. There were 6 males in each studied group. Frontal cortex, spinal cord, hippocampus, and cerebellum were isolated, stored at $-80^{\circ} \mathrm{C}$, and used in the subsequent experiments.

Total RNA was isolated from tissue samples according to manufacturer's protocol followed by reverse transcription, polymerase chain reaction and DNA electrophoresis in agarose gel. Reverse transcription reaction, the analysis of alternative splicing of exons 2, 3 (N-terminal part of tau protein) and exon 10 (C-terminal part) were performed, as described earlier [26]. The expression of tau-mRNA isoforms was expressed as the ratio of optical density band of tau isoform to optical density of $40 \mathrm{~S}$ ribosomal S12 protein RNA (housekeeping gene). Total tau-mRNA expression was expressed as a sum of 0,1 and $2 \mathrm{~N}$ isoforms expression. Each assay was repeated 2-4 times.

System UVI-KS4000, Syngen Biotechnology was used for densitometric analysis of RT-PCR. Results were expressed as means $\pm \mathrm{SD}$. Quantitative comparison between studied groups was performed by Student's $t$ test using Statistica 9.0 (StatSoft).

Studies were approved by the Ethic Committee for Experiments on Animals at the Medical University of Warsaw, Poland (no AO-KEZ/622/3/2008).

\section{Results}

\section{Total Tau-mRNA Expression}

The total tau-mRNA expression in frontal brain cortex of wild-type $(+/+)$ hybrids was $5.0 \pm 0.5,3.4 \pm 0.4$ and $3.0 \pm 0.2$ in mice age 70, 140 and 365 days, respectively (Fig. 1). In hippocampus of 70-day-old mice it was similar to the frontal cortex $(4.8 \pm 0.5)$, but in older groups it was higher and accounted for $4.4 \pm 0.3$ and $5.9 \pm 0.9$, in mice age 140 and 365 days, respectively (Fig. 1). The total taumRNA expression in spinal cord of wild-type mice age 70 
Fig. 1 Total tau-mRNA expression in various parts of the central nervous system. The total tau-mRNA was performed as a sum of 0,1 and $2 \mathrm{~N}$ isoforms expression, and expressed as a ratio of optical density bands of all tau isoforms to optical density of the housekeeping gene, as indicated in the Experimental Procedure section. filled diamond dashed lines - wild-type controls $(+/$ + ); filled triangle-Cra1/+ transgenics; filled squareSOD1/+ transgenics; filled circle-Cra1/SOD1 hybrides
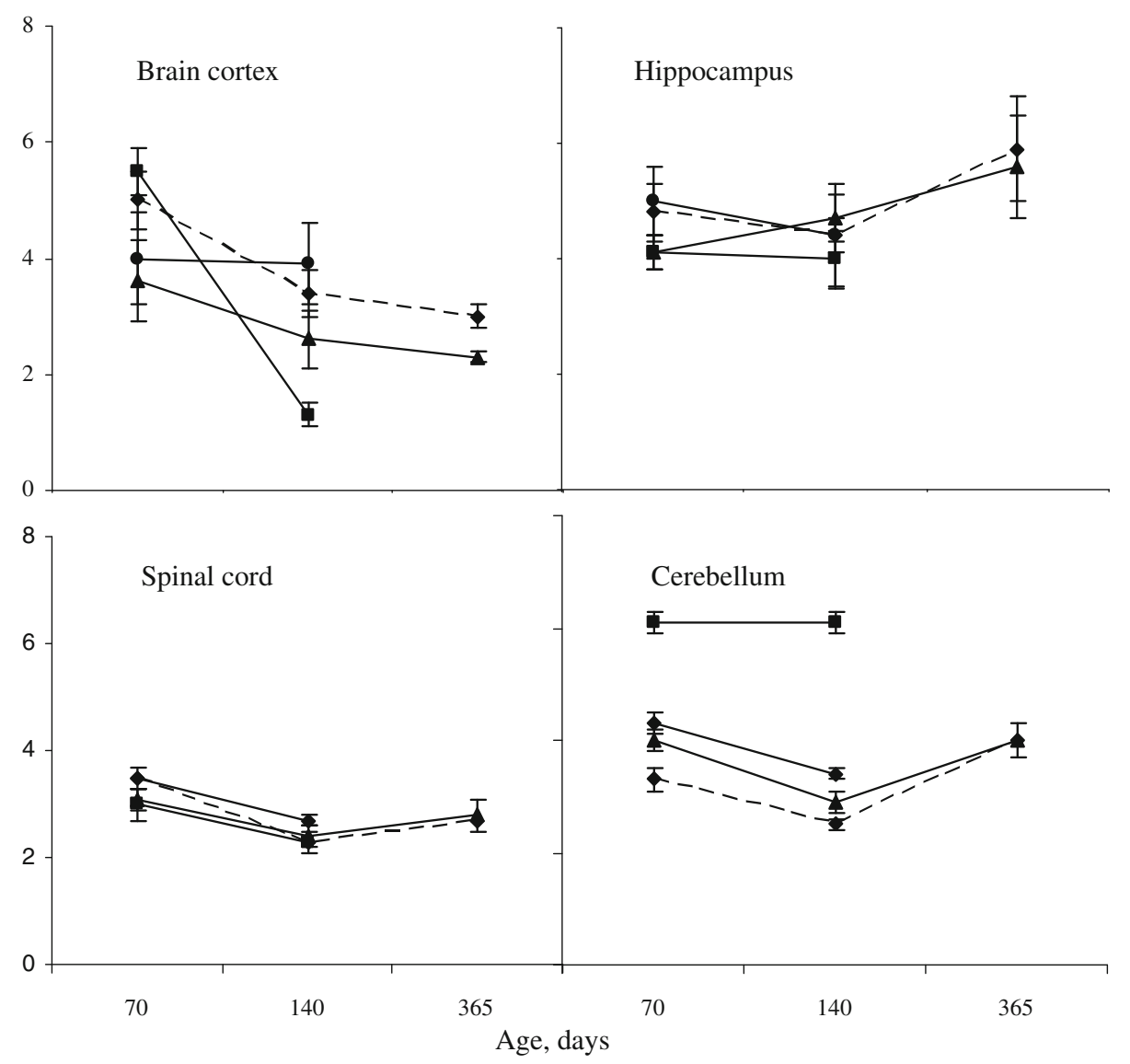

and 140 days was $3.5 \pm 0.3$ and $2.3 \pm 0.2$, and it was similar as in cerebellum, where it accounted for $3.3 \pm 0.7$ and $2.5 \pm 0.3$, respectively (Fig. 1). In 365-days-old groups it was $2.7 \pm 0.2$ and $4.0 \pm 0.3$, in spinal cord and cerebellum, respectively (Fig. 1).

In frontal brain cortex of Cra1/+ hybrids the total taumRNA expression was $3.6 \pm 0.7,2.6 \pm 0.5$ and $2.3 \pm 0.1$ in 70-, 140- and 365-day-old mice, respectively. In each age group it was significantly lower than in age-matched wild-type with $P<0.001$ for mice age 70 days, $P<0.05$ for 140-day-old, and $P<0.001$ for 365-day-old (Fig. 1). In SOD1/+ transgenics the expression was $5.5 \pm 0.4$ and $1.3 \pm 0.2$ in 70-day-old and 140-day-old group, respectively, and it was significantly decreased $(P<0.001)$ only in the older group (symptomatic stage of the disease). In Cra1/SOD1 hybrids age 70 days, tau expression accounted for $4.0 \pm 08$ and was significantly lower than in wild-type $(P<0.005)$, whereas in the older group it was unchanged $(3.9 \pm 0.7)$ (Fig. 1).

In hippocampus of Cra1/+ and SOD1/+ mice age 70 days tau expression was the same $(4.1 \pm 0.3)$ and it was lower than in control groups $(P<0.01)$, whereas in Cra1/ SOD1 hybrids it was unchanged $(5.0 \pm 0.6)$ (Fig. 1). Among other groups, slight decrease in the total tau expression was observed only in SOD1/+ group age 140 days $(P<0.01)$ (Fig. 1$)$.

In spinal cord of 70-day-old $\mathrm{Cra} 1 /+$ hybrids the total tau-mRNA expression was $3.1 \pm 0.2$, and it was slightly lower $(P<0.05)$ than in control group, whereas in older mice it was unchanged $(2.4 \pm 0.2$ in 140 - and $2.8 \pm 0.3$ in 365-day-old mice) (Fig. 1). In SOD1/+ group the total tau expression was significantly decreased only at presymptomatic stage $(3.0 \pm 0.2, P<0.01)$. In Cra1/SOD1 mice it was unchanged in group age 70 days, and higher than in wild-type control in the group age 140 days $(2.7 \pm 0.1$, $P<0.01$ ) (Fig. 1).

In cerebellum of Cra1/+ mice a slight increase in tau expression was observed only at age 70 days (4.0 \pm 0.4 , $P<0.05)$, whereas in older groups the expression was unchanged $(2.9 \pm 0.5$ in 140-day-old mice, $4.0 \pm 0.5$ in 365-day-old mice) (Fig. 1). Differently than in Cra1/+ groups, in SOD1/+ mice the total tau expression was significantly increased at both presymptomatic and symptomatic stages reaching more than 180 and $240 \%$ of the age-matched controls, respectively $(6.1 \pm 0.5$ in 70 -dayold mice and $6.1 \pm 0.7$ in 140-day-old mice, $P<0.001$ ) (Fig. 1). In Cra1/SOD1 transgenics the expression was also increased but not so much, and it accounted for $4.3 \pm 0.6$ 
in 70-day-old mice $(P<0.01)$ and $3.4 \pm 0.3$ in 140-dayold mice, $P<0.01$ ) (Fig. 1).

Alternative Splicing of Exons 2 and 3: Tau Isoforms Expression in Frontal Cortex

In 70-day-old Cra1/+ mice the expression of 0 and $1 \mathrm{~N}$ isoforms-mRNA in frontal cortex was the same as in wildtype control, and that of $2 \mathrm{~N}$ was significantly lower $(P<0.001)$ (Fig. 2). In older, but still presymptomatic Cra1/+ group (age 140 days), the expression of $0 \mathrm{~N}$ form was unchanged, $1 \mathrm{~N}$ was higher $(P<0.05)$, but $2 \mathrm{~N}$ lower
$(P<0.01)$ than in wild-type mice. In 365-day-old hybrids (symptomatic stage) the expression of all isoforms was significantly lower than in wild-type mice $(P<0.001$, $0.05,0.001$ for $0,1,2 \mathrm{~N}$, respectively). In SOD1/+ hybrids age 70 days, the expression of 0 and $1 \mathrm{~N}$ isoforms was higher than in wild type mice $(P<0.001)$, but the expression of $2 \mathrm{~N}$ was much lower $(P<0.001)$. In 140-day-old SOD1/+ mice (symptomatic stage) all isoforms expression was significantly lower $(P<0.001)$ than in control group. The profile of tau-mRNA isoforms expression in Cra1/SOD1 transgenics was partially like in Cra1/+ (0 N unchanged at age 70 and 140 days $)$, and
Fig. 2 Alternative splicing of exons 2 and 3 . The expression of the 0,1 and $2 \mathrm{~N}$ isoformmRNA was studied as indicated in the Experimental Procedure section. Black, solid bars; wildtype contols $(+/+)$; stripped bares-Cra1/+ mice; gray bares-SOD1/+ mice; dotted bares-Cra1/SOD1 hybrides. *changes statistically significant when compared with the wildtype control
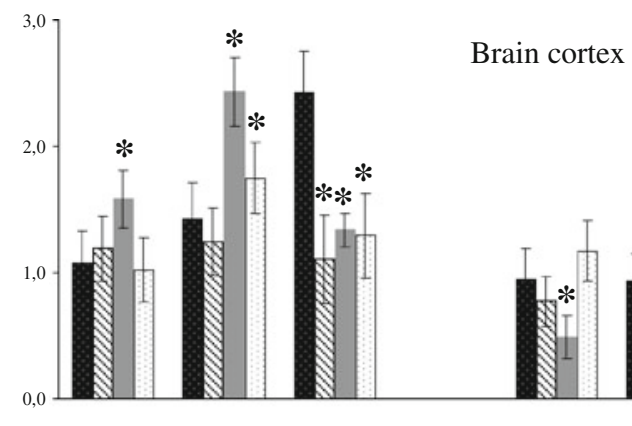$$
\text { , }
$$
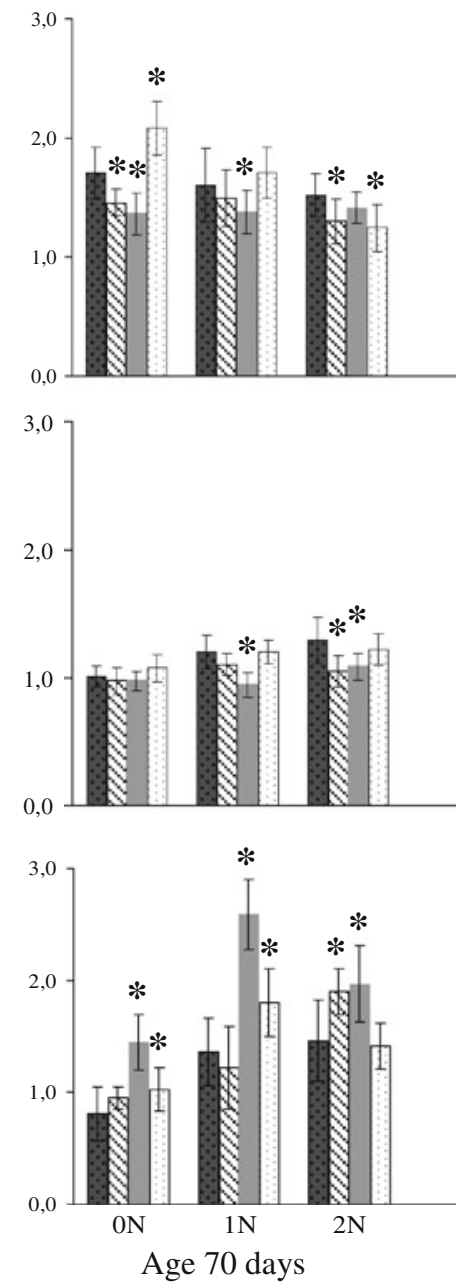

Spinal cord

Hippocampus
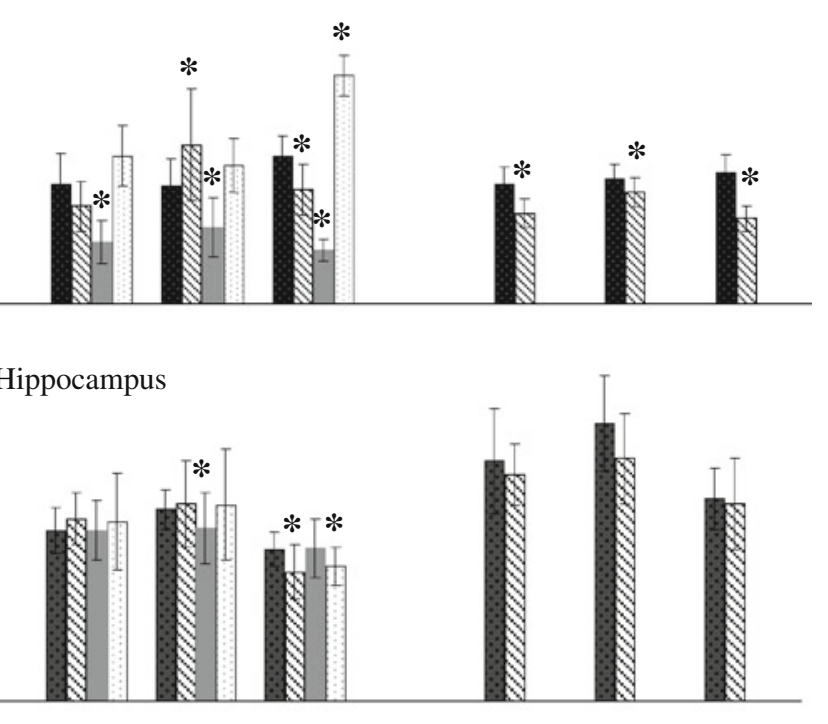
partially like in SOD1/+ group $(1 \mathrm{~N}$ increased at age 70 days, $P<0.05$ ). However, the expression of $2 \mathrm{~N}$ isoform in 140-day-old Cra1/SOD1 group was significantly increased $(P<0.001)$, whereas in Cra1/+ and SOD1/+ groups it was decreased (Fig. 2).

\section{Tau Isoforms Expression in Hippocampus}

In hippocampus of Cra1/+ presymptomatic mice (age 70 and 140 days) there was a slight decrease in the expression of $0 \mathrm{~N}$ and/or $2 \mathrm{~N}$ tau-mRNA isoforms $(P<0.05)$, but at symptomatic stage (age 365 days) the level of all isoforms expression was unchanged (Fig. 2). In SOD1/+ mice the expression of 0 and $1 \mathrm{~N}$ isoforms in the 70-day-old group (presymtomatic stage) and $1 \mathrm{~N}$ isoform in 140-day-old group (symptomatic stage) was slightly decreased $(P<0.05)$ (Fig. 2). In 70 -day-old group of Cra1/SOD1 mice, the $0 \mathrm{~N}$ expression was higher than in wild-type $(P<0.001)$, but in the older group (140-day-old), the expression of $2 \mathrm{~N}$ was decreased $(P<0.01)$ (Fig. 2).

\section{Tau Isoforms Expression in Spinal Cord}

In spinal cord of Cra1/+ transgenic mice at presymptomatic stage, the expression of 2 and $0 \mathrm{~N}$ isoform was decreased in younger (age 70 days) and older (age 140 days) group, respectively $(P<0.05$ and $P<0.005)$, whereas that of $1 \mathrm{~N}$ was increased in older mice $(P<0.001)$. At symptomatic stage (age 365 days), the $0 \mathrm{~N}$ isoform expression was significantly higher $(P<0.001)$ and $2 \mathrm{~N}$ lower $(P<0.001)$ than in control group (Fig. 2$)$. In SOD1/+ group age-70-days, the 1 and $2 \mathrm{~N}$ were decreased $(P<0.001$ and 0.005 , respectively), but in group of age-140-days, the expression of $2 \mathrm{~N}$ was higher (0.005) (Fig. 2). No differences on individual tau-mRNA isoforms expression were observed between the wild-type and transgenic mice in 70-day-old Cra1/SOD1 mice, but in 140-day-old group the expressions of 1 and $2 \mathrm{~N}$ were increased $(P<0.01$ and 0.001 , respectively) (Fig. 2).

\section{Tau-mRNA Isoforms Expression in Cerebellum}

In cerebellum of Cra1/+ mice, the expression of taumRNA isoforms was uniform in all studied groups (with the exception of slightly higher $2 \mathrm{~N}$ in 70-day-old transgenics, $P<0.01$ ), and it was similar as in age matched controls (Fig. 2). In SOD1/+ mice, differently than in other cns regions, the expression of all isoforms was much higher than in control groups, at both presymptomatic and symptomatic stages $(P<0.001)$ (Fig. 2$)$. In Cra1/SOD1 transgenics the expression of 0 and $1 \mathrm{~N}$ in 70-day-old mice was also increased $(P<0.05$ and $P<0.005)$, as well as the expression of 1 and $2 \mathrm{~N}$ in 140-day-old group

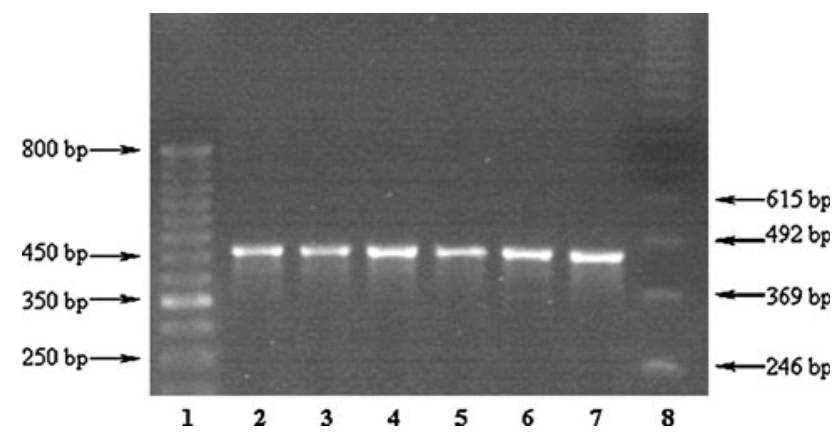

Fig. 3 Exon 10 splice variants in frontal cortex age 140 days. Lanes 1, 8-molecular weight markers (50 and $123 \mathrm{bp}$, respectively); lanes 2-5 mice age 140 days: lane 2-wild-type, lane 3-Cra1/+, lane 4SOD1/+, lane 5-Cra1/SOD1; lines 7, 8-wild-type and Cra1/+ transgenics age 365 days, respectively

$(P<0.05$ and $P<0.005)$, however, the increase was not as significant as in SOD1/+ groups (Fig. 2).

\section{Alternative Splicing of Exon 10}

The mRNA for exon 10 was present in all investigated tissues of wild-type controls, Cra1/+, SOD1/+ and Cra1/ SOD1 transgenics, at any studied age (Fig. 3).

\section{Discussion}

Tau is one of the main and most widely distributed microtubule-associated proteins in the central nervous system [27]. Mutations in the tau gene, located on chromosome 17, are responsible for frontotemporal dementia with parkinsonism (FTDP-17) indicating that tau dysfunction is sufficient to cause neurodegeneration [28, 29]. Tau polymorphisms are genetic risk factors for sporadic progressive supranuclear palsy (PSP) and corticobasal degeneration (CBD) [30]. In some pathological conditions tau protein undergoes post-translational modifications such as phosphorylation, ubiquitination, glycosylation, glycation [31-34]. Modified tau is an important component of neurofibrillary tangles in Alzheimer's disease, PSP, CDB, amyotrophic lateral sclerosis-parkinsonism dementia complex of Guam (ALS-G-PDC-G) and other tauopathies $[22,25,35]$. Aberrant aggregates of tau have been documented in most of the neurodegenerative diseases with filamentous inclusions [27, 36].

Amyotrophic lateral sclerosis (ALS) is the most devastating condition among neurodegenerative diseases. The majority of ALS cases are sporadic of still unknown etiology and approximately $10 \%$ are familial [37]. Approximately $20 \%$ of familial ALS are associated with mutations in the gene encoding for SOD1. However, the exact mechanisms by which mutations in the SODl gene 
contribute to pathogenesis of ALS remain elusive [38]. Despite this fact, transgenic mice carrying a human mutant SOD1 became an animal model for ALS [9, 39]. In our previous studies we have indicated that the SOD1G93A mutation influences alternative splicing and the total taumRNA expression in a mouse central nervous system (strain C57BL/6SJ) [26]. The changes were prominent in frontal cortex, but not in hippocampus and spinal cord. In frontal cortex at symptomatic stage of the disease, the level of total tau mRNA was decreased by about $20 \%$, mainly because of $2 \mathrm{~N}$ isoform down-regulation [26, 40]. In the present studies conducted on $\mathrm{B} 6-\mathrm{C} 3 \mathrm{H}$ hybrids with the SOD1G93A mutation (SOD1/+) the total tau expression in frontal cortex at symptomatic stage was even lower (decreased by more than 60\%) and the level of all isoforms was decreased, but especially that of $2 \mathrm{~N}$ (a threefold decrease). The total tau-mRNA expression in frontal cortex of mice with the Dynclhl mutation (Cra1/+ hybrids) was also decreased but only by $25-30 \%$. It was observed in all Cra1/+ groups, but was independent of their age and clinical stage. In hippocampus and spinal cord of both SOD1/+ and Cra1/+ transgenics slight changes were observed only at presymptomatic stage (10-15\% decrease). Since hippocampus is not affected in ALS/ MND, the results obtained for SOD1/+ transgenics are understandable. However, the lack or only mild changes observed in spinal cord, the structure primarily affected by the neurodegenerative process, is puzzling. The changes in tau expression are expected to occur mainly within the motor neurons, which - unlike in the frontal cortex-form only a small part of the spinal cord volume. When recalculating the results for the total tissue volume, the observed changes may be too low to influence the final out-come. Detailed immunohistochemical studies could potentially solve this question. Beside the possible method limitations, the results leave open the question on the low tau involvement in the degeneration of the alpha-motor neurons bodies.

Practically no changes in the total tau-mRNA and its isoforms expression in cerebellum of $\mathrm{Cra} 1 /+$ transgenics were observed. Unlike in Cra1/+ in SOD1/+ mice the total tau expression was almost 2- and over twofold higher at presymptomatic and symptomatic SOD1/+ hybrids, respectively. The levels of all isoforms were elevated at both stages. The present results are with agreement with our earlier studies conducted on SOD1G93A mice (which were not hybrids with $\mathrm{C} 3 \mathrm{H}$ ). We have shown that the level of total tau-mRNA increases in cerebellum already in newborns (by 25\%) and it further rises with age reaching the highest value at symptomatic stage (by $40 \%$ ) due to enhance of all isoforms expression [40]. Cerebellum is the structure usually not studied in motor neuron diseases since cerebellar dysfunction is not a clinical feature of ALS.
However, an increasing body of evidence shows the involvement of extra-motor neuron regions in the pathology of this disorder. Chung et al. [41, 42] detected the presence of astrocytes immunoreactive for phosphorylated extracellular signal-regulated kinases (pERK) and glycogen synthase kinase (GSK) 3alpha involved in anti-apoptotic signal transduction pathways in the spinal cord, brainstem, central gray and cerebellar nuclei of symptomatic SODG93A transgenic mice. A recent neuropathological study performed in humans with ALS showed neuronal and glial TDP-43 pathology not only in cortical areas but also in the nigro-striatal system and cerebellum [43]. The above findings suggests that ALS does not selectively affect the pyramidal motor system, but that it is a multisystem neurodegenerative TDP-43 proteinopathy. The functional MRI showed enhanced activation of bilateral motor areas and ipsilateral cerebellum in response to task performance in patients with ALS compared to healthy controls. Performing the task resulted in additional activation of contralateral cerebellum and bilateral capsula interna in ALS patients [44]. Another fMRI study suggests that functional compensation following the degeneration of cortical and spinal motor neurons in ALS leads to a recruitment of subcortical motor structures, including supplementary motor area, basal ganglia, brainstem, and cerebellum [45]. Although the above results do no explain the differences of tau expression between cerebellum and the structures stereotypically involved in ALS pathogenesis, they indicate the presence of neural reorganization and the possibility of functional compensation in this disease. More research is needed to elucidate this phenomenon, the more that our unpublished data indicate significant changes in the expression of some motor proteins in cerebellum compared to brain cortex or spinal cord.

In Cra1/SOD1 double heterozygotes there were less changes in individual isoforms tau expression than in Cra1/ + or SOD1/+ transgenics. In frontal cortex the changes resembled the results obtained in presymptomatic SOD1/+ group, whereas in hippocampus they looked rather like in Cra1/+ mice, at both presymptomatic and symptomatic stages. Few changes in tau-mRNA isoforms expression were noticed in spinal cord. In cerebellum, an increase in tau isoforms was observed, but not as significant as in SOD1/+ groups. The slight changes in tau-isoforms expression resulted in unchanged total tau-mRNA levels or lead to only mild changes (mainly in cerebellum). The lack of tau expression changes in this model goes along with the finding of Kieran et al. [10] who have shown the restoration of retrograde transport in the double transgenic model. It may at least partially explain better clinical condition and longer survival time of mice carrying both dynein heavy chain 1 and SODI mutations compared to those with the SOD1 mutation alone [11]. 
Tau isoforms $(0,1,2 \mathrm{~N})$ differ from each other by amino acids sequence at the $\mathrm{N}$-terminal part of protein. The $2 \mathrm{~N}$ isoform is the longest since it contains an insert of 58 amino acid residues encoded by exons 2 and 3. The $1 \mathrm{~N}$ isoform contains an insert of 29 amino acids encoded by exons 2 , and the shortest $0 \mathrm{~N}$ isoform does not contain products of exons 2 and 3 [21]. N-terminal part of tau protein is essential for tau function, since it is involved in signal transduction pathways. It interacts with src-family tyrosine kinases, phospholipase C-gamma isoenzymes, as well as with other elements of cytoskeleton and neural plasma membrane [24, 46, 47]. It is also crucial for the stabilization and organization of axons and determining spacing between their microtubules [48, 49]. Therefore, the alternative splicing of tau exons 2 and 3 may alter the diameter of axons [50]. The changes we observed, were most prominent in frontal cortex and cerebellum of SOD1/+ and, to a lower extent, in Cra1/+ transgenics. The big differences, especially in $2 \mathrm{~N}$ isoform-mRNA expression, at symptomatic stage of Cra1/+ and SOD1/+ transgenics (about 75 and $30 \%$ decrease, respectively), as well as spectacular differences in all $\mathrm{N}$-terminal isoforms expression in cerebellum (no changes in Cra/+ and dramatic increase in SOD1/+ mice) indicate distinct molecular mechanisms of dynein heavy chain- and SOD1-induced motor neuron degeneration. Since the role of its N-terminal forms individual tau isoforms is not known, it is not easy to understand the pathological consequences caused by the disturbances observed in their expression. It is known that tau filaments, but not monomeric soluble tau forms, selectively inhibit anterograde (kinesin-dependent) fast axonal transport, triggering the release of conventional kinesin from axoplasmic vesicles. LaPointe et al. [51] indicated that first 18 amino acids at the N-terminus tau of squid axoplasm are required to elicit the inhibitory effect of tau filaments on fast axonal transport. The longest tau isoform (441 amino acids, hTau40) shows greater propensity to form filaments than the shorter ones [52]. Therefore, the increased expression of all N-terminal isoforms in cerebellum but not in other cns regions of SOD1/+ transgenics may lead to formation of tau aggregates. Till now little is known about the role of the $\mathrm{N}$-terminal tau domain in neuronal survival. The studies conducted by Amadoro et al. [53] on adenovirally mediated expression of tau's N-terminal domains indicate, that the exogenous overexpression of the N-terminal (1-230) as well as full-length tau (1-441) inhibit the onset of apoptosis, whereas the overexpression of the short fragment [1-44] exerts a toxic effect leading to a necrotic-type death of neurons. At present it is difficult to say, whether the disturbances in tau N-terminal isoforms-mRNA expression, observed in our studies, are connected with higher (in brain cortex) or lower (in cerebellum) sensitivity to SOD1-related neurodegeneration.
Besides, Cra1 mice show defects in dynein complex resulting in an impaired retrograde axonal transport [6-8]. This impairment may be due to tau dysfunction caused by defective N-terminal alternative splicing, since the tau N-terminal domain enhances the attachment of dynein/ dynactin complex to microtubules [19]. Such mechanism may occur in brain cortex where, as we showed, the expression of all three tau isoforms decreases at symptomatic stage, but not in cerebellum where it remains unchanged.

Thus, both Dynclhl and SOD1G93A mutations cause changes in tau-mRNA expression, which may subsequently affect the structure of cytoskeleton and axonal transport. However, clear differences between tau expression in Cra1/ + and SOD1/+ transgenic animals indicate a distinct pathogenic entity of these two conditions.

Acknowledgments This work was supported by grants NN401 417436 and NN401 063436 from the Ministry of Science and Higher Education of Poland, and 1WK/N/2010 from the Medical University of Warsaw, Poland.

Open Access This article is distributed under the terms of the Creative Commons Attribution Noncommercial License which permits any noncommercial use, distribution, and reproduction in any medium, provided the original author(s) and source are credited.

\section{References}

1. El-Kadi AM, Soura V, Hafezparast M (2007) Defective axonal transport in motor neurodisease. J Neurosci Res 85:2557-2566

2. Gibbons IR (1995) Dynein family of motor proteins: present status and future questions. Cell Motil Cytoskeleton 32:136-144

3. Goldstein LS, Yang Z (2000) Microtubule-based transport systems in neurons: the roles of kinesins and dyneins. Annu Rev Neurosci 23:39-71

4. Samso M, Radermacher M, Frank J et al (1988) Structural characterization of a dynein motor domain. J Mol Biol 276:927-937

5. Harada A, Takei Y, Kanai Y et al (1998) Golgi vesiculation and lysosome dispersion in cells lacking cytoplasmic dynein. J Cell Biol 141:51-59

6. Hrabe de Angelis MH, Flaswinkel H, Fuchs H et al (2000) Genome-wide, large-scale production of mutant mice by ENU mutagenesis. Nat Genet 25:444-447

7. LaMonte BH, Wallace KE, Holloway BA et al (2002) Disruption of dynein/dynactin inhibits axonal transport in motor neurons causing late-onset progressive degeneration. Neuron 34:715-727

8. Hafezparast M, Klocke R, Ruhrberg C et al (2003) Mutations in dynein link motor neuron degeneration to defects in retrograde transport. Science 300:808-881

9. Gurney ME, Pu H, Chiu AY et al (1994) Motor neuron degeneration in mice that express a human $\mathrm{Cu}, \mathrm{Zn}$ superoxide dismutase mutation. Science 264:1772-1775

10. Kieran D, Hafezparast M, Bohnert S et al (2005) A mutation in dynein rescues axonal transport defect and extends the life span of ALS mice. J Cell Biol 169:561-567

11. Teuchert M, Fischer D, Schwalenstoecker B et al (2006) A dynein mutation attenuates motor neuron degeneration in SOD1G93A mice. Exp Neurol 198:271-274 
12. Chen X-J, Eleni N, Levedakou KJ et al (2007) Proprioceptive sensory neuropathy in mice with a mutation in the cytoplasmic dynein heavy chain 1 gene. J Neurosci 27:14515-14524

13. Ilieva HS, Yamanaka K, Malkumus S et al (2008) Mutant dynein (Loa) triggers proprioceptive axon loss that extends survival only in the SOD1 ALS model with highest motor neuron death. PNAS 105:12599-12604

14. Dupuis L, Fergani A, Braunstein KE et al (2009) Mice with a mutation in the dynein heavy chain 1 gene display sensory neuropathy but lack motor neuron disease. Exp Neurol 215:146-152

15. Braunstein KE, Eschbach J, Pona-Voros K et al (2010) A point mutation in the dyein heavy chain gene leads to striatal atrophy and compromises neurite outgrowht of structal neurons. Hum Mol Genet 19:4385-4398

16. Weingarten MD, Lockwood AH, Hwo SY et al (1975) A protein factor essential for microtubule assembly. Proc Natl Acad Sci USA 72:1858-1862

17. Hirokawa N, Shiomura Y, Ogabe S (1988) Tau proteins: the molecular structure and mode of binding on microtubules. J Cell Biol 107:1449-1459

18. Schoenfeld TA, Obar BA (1994) Diverse distribution and function of fibrous microtubule-associated proteins in the nervous system. Int Rev Cytol 151:67-137

19. Magnani E, Fan J, Gasparini L, Golding M et al (2007) Interaction of tau protein with the dynactin complex. EMBO J 26: 4546-4554

20. Puls I, Oh SJ, Sumner CJ et al (2005) Distal spinal and bulbar muscular atrophy caused by dynactin mutation. Ann Neurol 57:687-694

21. Andreadis A, Brown WM, Kosik KS (1992) Structure and novel exons of the human tau gene. Biochemistry 31:10626-10633

22. Poorkaj P, Kas A, D'Souza I et al (2001) A genomic sequence analysis of the mouse and human microtubule-associated protein tau. Mamm Genome 12:700-712

23. Goedert M, Spillantini MG, Crowther RA (1992) Cloning of a big tau microtubule associated protein characteristic of the peripheral nervous system. Proc Natl Acad Sci USA 89:1983-1987

24. Lee G, Neve RL, Kosik KS (1989) The microtubule binding domain of tau protein. Neuron 2:1615-1624

25. Goedert M, Spillantini MG, Jakes R et al (1989) Multiple isoforms of human microtubule-associated protein tau: sequences and localization in neurofibrillary tangles of Alzheimer's disease. Neuron 3:519-526

26. Usarek E, Kuźma-Kozakiewcz M, Schwalenstöcker B et al (2006) Tau isoforms expression in transgenic mouse model of amyotrophic lateral sclerosis. Neurochem Res 31:597-602

27. Robert M, Mathuranath PS (2007) Tau and tauopathies. Neurol India 27:11-16

28. Hutton M, Lendon CL, Rizzu P et al (1998) Association of missense and $5^{\prime}$-splice-site mutations in tau with the inherited dementia FTDP-17. Nature 393:702-705

29. Yancopoulou D, Spillantini MG (2003) Tau protein in familial and sporadic diseases. Neuromol Med 4:37-48

30. Cairns NJ, Lee VM, Trojanowski JQ (2004) The cytoskeleton in neurodegenerative diseases. J Pathol 204:438-449

31. Grundke-Iqbal I, Iqbal K, Tung YC et al (1986) Abnormal phosphorylation of the microtubule-associated protein tau in Alzheimer cytoskeletal pathology. Proc Natl Acad Sci USA 83:4913-4917

32. Mori H, Kondo J, Ihara $Y$ et al (1987) Ubiquitin is a component of paired helical filaments in Alzheimer's disease. Science 235: 1641-1644

33. Wang JZ, Grundke-Iqbal I, Iqbal K (1996) Glycosylation of micro-tubule-associated protein tau: an abnormal posttranslational modification in Alzheimer's diseases. Nat Med 2:871-875
34. Nacharaju P, Ko L, Yen SH (1997) Characterization of in vitroglycation sites of tau. J Neurochem 69:1709-1719

35. Buee L, Bussiere T, Buee-Scherrer V et al (2000) Tau protein isoforms, phosphorylation and role in neurodegenerative disorders. Brain Res Brain Res Rev 33:95-130

36. Spillantini MG, Goedert M (1998) Tau protein pathology in neurodegenerative diseases. Trends Neurosci 21:428-433

37. Mulder DW, Kurland LT, Offord KP et al (1986) Familial adult motor neuron disease: amyotrophic lateral sclerosis. Neurology 36 : $511-517$

38. Kuzma-Kozakiewicz M, Kwiecinski H (2011) New therapeutic targets for amyotrophic lateral sclerosis. Expert Opin Ther Targets 15:127-143

39. Tuy PH, Raju P, Robinson KA et al (1996) Transgenic mice carrying a human mutant superoxide dismutase transgene develop neuronal cytoskeletal pathology resembling human amyotrophic lateral sclerosis lesions. Proc Natl Acad Sci USA 93:3155-3160

40. Barańczyk-Kuźma A, Usarek E, Kuźma-Kozakiewcz M et al (2007) Age-related changes in tau expression in transgenic mouse model of amyotrophic lateral sclerosis. Neurochem Res 32: $415-421$

41. Chung YH, Joo KM, Lim HC et al (2005) Immunohistochemical study on the distribution of phosphorylated extracellular signalregulated kinase (ERK) in the central nervous system of SOD1G93A transgenic mice. Brain Res 19:203-209

42. Chung YH, Joo KM, Kim DJ et al (2008) Immunohistochemical study on the distribution of glycogen synthase kinase 3alpha in the central nervous system of SOD1(G93A) transgenic mice. Neurol Res 30:926-931

43. Geser F, Brandmeir NJ, Kwong LK et al (2008) Evidence of multisystem disorder in whole-brain map of pathological TDP-43 in amyotrophic lateral sclerosis. Arch Neurol 65:636-641

44. Han J, Ma L (2006) Functional magnetic resonance imaging study of the brain in patients with amyotrophic lateral sclerosis. Chin Med Sci J 21:228-233

45. Konrad C, Jansen A, Henningsen H et al (2006) Subcortical reorganization in amyotrophic lateral sclerosis. Exp Brain Res 172:361-369

46. Lee G, Newman ST, Gard DL et al (1998) Tau interacts with srcfamily non-receptor tyrosine kinases. J Cell Sci 111:3167-3177

47. Hwang SC, Jhon DY, Bae YS et al (1996) Activation of phospholipase C-gamma by the concerted action of tau proteins and arachidonic acid. J Biol Chem 271:8313-8320

48. Brandt R, Leger J, Lee G (1995) Interaction of tau with the neural plasma membrane mediated by tau's amino-terminal projection domain. J Cell Biol 131:1327-1340

49. Georgieff IS, Liem RK, Couchie D et al (1993) Expression of high molecular weight tau in the central and peripheral nervous systems. J Cell Sci 105:729-737

50. Chen J, Kanai Y, Cowan NJ, Hirokawa N (1992) Projection domains of MAP2 and tau determine spacings between microtubules in dendrites and axons. Nature 360:674-677

51. LaPointe NE, Morfini G, Pigino G et al (2009) The amino terminus of tau inhibits kinesin-dependent axonal transport: implications for filament toxicity. J Neurosci Res 87:440-451

52. Yang W, Sopper MM, Leystra-Lantz C, Strong MJ (2003) Microtubule-associated tau protein positive neuronal and glial inclusions in ALS. Neurology 61:1766-1773

53. Amadoro G, Serafino AL, Barbato C et al (2004) Role of $\mathrm{N}$-terminal tau domain integrity on the survival of cerebellar granule neurons. Cell Death Differ 11:217-230 